\title{
miRNA profiling for the early detection and clinical monitoring of diabetic kidney disease
}

\author{
"The implementation of miRNA profiling in the clinical management \\ of diabetic kidney disease has the potential to dramatically alter \\ patient outcomes."
}

First draft submitted: 27 October 2017; Accepted for publication: 21 November 2016; Published online: 18 January 2017

Keywords: biomarkers $\bullet$ diabetes $\bullet$ diabetic kidney disease $\bullet$ estimated glomerular filtration rate $\bullet$ exosomes $\bullet$ kidney $\bullet$ microalbuminuria $\bullet$ miRNAs $\bullet$ noncoding RNA $\bullet$ renal function

\section{Biomarkers for the early detection of diabetic kidney disease}

Diabetic kidney disease underlies most of the reduced life expectancy in diabetic patients [1]. The annual incidence of diabetic kidney disease has more than doubled in the past decade, and today accounts for nearly $50 \%$ of all end-stage renal disease (ESRD) cases [2]. In individuals with ESRD, renal replacement therapy is used to control uremia and other metabolic consequences of kidney failure, and yet $<50 \%$ of patients are still alive 5 years post diagnosis [3]. Poor clinical outcomes for the diabetic kidney disease patients are due, in part, to the delayed presentation of symptoms, which often only become apparent $5-10$ years after the actual initiation of renal damage. Thus, a major need facing the clinical management of diabetic kidney disease is early detection of disease, to attenuate loss of renal function and improve patient outcomes.

Diabetic kidney disease is a progressive microvascular complication of diabetes caused by damage to the capillaries of the glomeruli, and is characterized by persistent albuminuria $(>300 \mathrm{mg} /$ day or $>200 \mu \mathrm{g} /$ $\mathrm{min}$ ), progressive decline in glomerular filtration rate (GFR), and elevated arterial blood pressure. Incremental changes in urinary albumin excretion (UAE) rates are corre- lated with the development of diabetic kidney disease and subsequent progression to clinically severe forms of renal impairment. Until recently, the disease was categorized according to stages of microalbuminuria (UAE $>20 \mu \mathrm{g} / \mathrm{min}$ and $\leq 199 \mu \mathrm{g} / \mathrm{min}$ ) and macroalbuminuria (UAE $\geq 200 \mu \mathrm{g} / \mathrm{min}$ ). Although microalbuminuria has been the most widely accepted measure of early renal impairment in diabetes, its use as a clinical marker for the diagnosis and prognosis of diabetic kidney disease is limited. Notably, microalbuminuria is not a specific marker for diabetic kidney disease, but instead reflects changes in the glomerulus resulting from any kind of chronic kidney disease. In addition, UAE rates are oftentimes within normal range in diabetic patients with an estimated GFR indicative of chronic kidney disease $[4,5]$. While microalbuminuria has been considered a risk factor for macroalbuminuria, not all patients progress to this stage, and a significant proportion of patients even regress to normoalbuminuria [6,7]. Early studies reported that approximately $80 \%$ of diabetic patients with microalbuminuria progressed to proteinuria over 6-14 years [8]; however, more recent work showed that only $30-45 \%$ of microalbuminuric patients progress to overt nephropathy over a similar period of time [9], likely due to more intensive

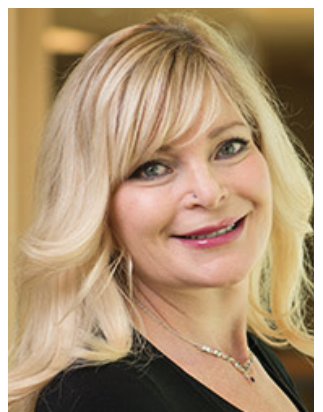

Johanna K DiStefano Department of Biomedical Research, Center for Genes, Environment, and Health, National Jewish Health, 1400 Jackson Street, Denver, CO 80206, USA

Tel.: +1 3032702357

distefanoj@njhealth.org 
interventions to manage hyperglycemia and hypertension. Finally, substantial renal damage, resulting from changes in the glomerular basement membrane and effects of inflammation, has already occurred in many patients prior to the clinical manifestation of microalbuminuria [6,10]. Taken together, these studies indicate that progression of diabetic kidney disease, represented as a decline in GFR, does not necessarily parallel increasing rates of UAE.

"In general, miRNAs are protected from degradation through encapsulation in microvesicles, such as exosomes, or binding with circulating proteins."

Thus, for some diabetic patients, microalbuminuria is neither an accurate marker of renal dysfunction, nor a reliable predictor of disease progression. Because methods are not yet available to identify those patients at risk for developing microvascular complications before detrimental changes actually occur, markers to detect disease at an early stage of renal impairment would provide enormous clinical benefit in preventing loss of kidney function. A number of urinary proteins have emerged as potential markers of early renal injury in diabetic kidney disease, although widespread clinical implementation of these molecules currently awaits further validation.

\section{miRNAs as potential biomarkers for renal impairment in diabetes}

Over the past several years, a new class of potential biomarkers, miRNAs, has shown promise for predicting disease status. These molecules are endogenous, singlestranded RNAs that function primarily to regulate gene expression by binding to complementary sequences on mRNA target genes [11]. miRNAs are involved in a broad range of biological processes such as hematopoietic development, apoptosis, proliferation, modulation of immune response, insulin secretion, and cellular differentiation [12]. Given this extensive involvement, it is not surprising that dysregulation of miRNAs has been shown to impact the development of many different diseases, including cancer, neurological disorders, diabetes, complications of diabetes and others [13].

Recent progress in miRNA research has not only provided an enhanced understanding of the complexity of the pathophysiology of kidney diseases, but has also laid the foundation for the identification of new diagnostic biomarkers. For example, many studies in various renal diseases have shown that miRNA expression is differentially regulated, and as such, it is possible that 'molecular signature profiles' might be developed to inform disease diagnosis and risk of progression $[14,15]$. Urinary profiling in diabetic patients identified levels of 27 miRNAs that varied according to stage of untreated nephropathy [16]. Further analysis showed that miRNA signatures could predict the development of microalbuminuria at least 2 years before it occurred [17]. Examination of plasma levels of five TGFB1-regulated miRNAs in two prospective groups of diabetes patients, including progressors (individuals with proteinuria and normal renal function who rapidly progressed with a fast rate of estimated GFR decline) and nonprogressors (individuals with proteinuria with normal renal function who maintained normal renal function) found that two miRNAs, let-7c-5p and miR-29a-3p, were associated with protection against rapid progression, while two others, let-7b-5p and miR-21-5p, were associated with increased risk of ESRD [18]. These studies, combined with others $[19,20]$, provide good evidence that urinary and circulating levels of miRNAs are dysregulated at an early stage in diabetic patients at risk for progression to ESRD.

miRNAs are present in many biological fluids, including blood, serum, and urine [21], and changes in levels of circulating miRNAs in these biofluids have been associated with different renal diseases $[15,22,23]$. In general, miRNAs are protected from degradation through encapsulation in microvesicles, such as exosomes, or binding with circulating proteins. Urine is rich in exosomes, which are secreted by cells from all nephron segments and carry protein, mRNA, and miRNA markers of renal dysfunction and structural injury [24]. Due to mechanical and charge barriers in the glomerulus, circulating microvesicles from serum cannot cross the nephron, suggesting that urinary exosomes emanate primarily from renal cells [25]. Content from urinary exosomes, therefore, has significant potential to reveal a comprehensive snapshot of overall renal health [26]. Importantly, changes in the levels of urinary exosome-derived miRNAs have been correlated with early renal impairment in patients with T2D [19] and progression to fibrosis in lupus nephritis [27].

\section{Next steps for miRNA biomarker development \& translation to clinical settings}

miRNAs demonstrate sensitivity, specificity, concentration responsiveness to disease pathology and translatability between preclinical and clinical settings [28]. However, the role of miRNAs as potential biomarkers for renal impairment remains in the discovery stage. Among the studies reported thus far, there is a little overlap in findings, which may reflect differences in design, experimental approach, and method of analysis. In addition, individual variability, including race, 
gender, environmental exposures, and dietary components, contribute to variance in miRNA levels [29]. Before translation of miRNA profiling into clinical practice, studies using large, carefully phenotyped cohorts will be necessary to identify and validate miRNA profiles that consistently and accurately reflect disease status. Baseline parameters, such as assessment of intra- and inter-individual variability, standardization of methods for all steps of sample collection and processing, RNA extraction, miRNA quantification, and analysis, will also need to be established to minimize interlaboratory biases. Once consensus procedures have been defined for miRNA profiling, interpretation and comparison of different study results will be more straightforward. At that point, use of miRNA biomarkers will need to demonstrate enhanced performance in disease diagnosis, and subsequent improvements in targeted clinical outcomes, compared with current diagnostic standards.

At present, renal indicators of kidney damage and progression are limited to the analysis of urinary proteins and changes in GFR. Circulating and urinary miRNAs show promise as biomarkers for the improved diagnosis, prognosis, and clinical monitoring of diabetic kidney disease. Once issues related to discovery

\section{References}

1 Afkarian M, Sachs MC, Kestenbaum B et al. Kidney disease and increased mortality risk in Type 2 diabetes. J. Am. Soc. Nephrol. 24(2), 302-308 (2013).

2 Kanwar YS, Sun L, Xie P, Liu FY, Chen S. A glimpse of various pathogenetic mechanisms of diabetic nephropathy. Annu. Rev. Pathol. 6, 395-423 (2011).

3 Blumenthal SS. Evolution of treatment for diabetic nephropathy: historical progression from RAAS inhibition and onward. Postgrad. Med. 123(6), 166-179 (2011).

4 Kramer HJ, Nguyen QD, Curhan G, Hsu CY. Renal insufficiency in the absence of albuminuria and retinopathy among adults with Type 2 diabetes mellitus. JAMA 289(24), 3273-3277 (2003).

5 Perkins BA, Nelson RG, Ostrander BE et al. Detection of renal function decline in patients with diabetes and normal or elevated GFR by serial measurements of serum cystatin $\mathrm{C}$ concentration: results of a 4-year follow-up study. J. Am. Soc. Nephrol. 16(5), 1404-1412 (2005).

6 Perkins BA, Ficociello LH, Roshan B, Warram JH, Krolewski AS. In patients with Type 1 diabetes and newonset microalbuminuria the development of advanced chronic kidney disease may not require progression to proteinuria. Kidney Int. 77(1), 57-64 (2010).

7 Perkins BA, Ficociello LH, Silva KH, Finkelstein DM, Warram JH, Krolewski AS. Regression of microalbuminuria in Type 1 diabetes. N. Engl. J. Med. 348(23), 2285-2293 (2003). and standardization of methods for biomarker evaluations, which is an area of active research in the field of oncology, are overcome, miRNA profiling may provide an alternative approach for the early detection of renal impairment in diabetes. The implementation of miRNA profiling in the clinical management of diabetic kidney disease has the potential to dramatically alter patient outcomes. Compared with the current gold standard of tissue biopsy, which is invasive, prone to sampling error, expensive, and cannot be performed at early stages of disease when aggressive treatments to prevent progression might be initiated, miRNA biomarkers offer a noninvasive and cost-effective means to detect changes in renal metabolism long before significant damage occurs.

\section{Financial \& competing interests disclosure}

The author has no relevant affiliations or financial involvement with any organization or entity with a financial interest in or financial conflict with the subject matter or materials discussed in the manuscript. This includes employment, consultancies, honoraria, stock ownership or options, expert testimony, grants or patents received or pending, or royalties.

No writing assistance was utilized in the production of this manuscript.

8 Mogensen CE, Christensen CK. Predicting diabetic nephropathy in insulin-dependent patients. $N$. Engl. J. Med. 311(2), 89-93 (1984).

9 Rossing P, Hougaard P, Parving HH. Progression of microalbuminuria in Type 1 diabetes: ten-year prospective observational study. Kidney Int. 68(4), 1446-1450 (2005).

10 Fioretto P, Steffes MW, Mauer M. Glomerular structure in nonproteinuric IDDM patients with various levels of albuminuria. Diabetes 43(11), 1358-1364 (1994).

11 Kim DH, Saetrom P, Snove O Jr, Rossi JJ. MicroRNAdirected transcriptional gene silencing in mammalian cells. Proc. Natl Acad. Sci. USA 105(42), 16230-16235 (2008).

12 Chang TC, Mendell JT. microRNAs in vertebrate physiology and human disease. Annu. Rev. Genomics. Hum. Genet. 8, 215-239 (2007).

13 Alvarez ML, Distefano JK. The role of noncoding RNAs in diabetic nephropathy: potential applications as biomarkers for disease development and progression. Diabetes. Res. Clin. Pract. 99(1), 1-11 (2013).

14 Gottardo F, Liu CG, Ferracin M et al. Micro-RNA profiling in kidney and bladder cancers. Urol. Oncol. 25(5), 387-392 (2007).

15 Neal CS, Michael MZ, Pimlott LK, Yong TY, Li JY, Gleadle JM. Circulating microRNA expression is reduced in chronic kidney disease. Nephrol. Dial. Transplant. 26(11), 3794-3802 (2011).

16 Argyropoulos C, Wang K, McClarty S et al. Urinary microRNA profiling in the nephropathy of Type 1 diabetes. PLoS ONE 8(1), e54662 (2013). 
17 Argyropoulos C, Wang K, Bernardo J et al. Urinary microRNA profiling predicts the development of microalbuminuria in patients with Type 1 diabetes. J. Clin. Med. 4(7), 1498-1517 (2015).

18 Pezzolesi MG, Satake E, McDonnell KP, Major M, Smiles AM, Krolewski AS. Circulating TGF-beta1-regulated miRNAs and the risk of rapid progression to ESRD in Type 1 diabetes. Diabetes 64(9), 3285-3293 (2015).

19 Delic D, Eisele C, Schmid R et al. Urinary exosomal miRNA signature in Type II diabetic nephropathy patients. PLoS ONE 11(3), e0150154 (2016).

20 Skupien J, Warram JH, Smiles AM et al. The early decline in renal function in patients with Type 1 diabetes and proteinuria predicts the risk of end-stage renal disease. Kidney Int. 82(5), 589-597 (2012).

21 Cortez MA, Calin GA. MicroRNA identification in plasma and serum: a new tool to diagnose and monitor diseases. Expert. Opin. Biol. Ther. 9(6), 703-711 (2009).

22 Hauser S, Wulfken LM, Holdenrieder S et al. Analysis of serum microRNAs (miR-26a-2*, miR-191, miR-337-3p and miR-378) as potential biomarkers in renal cell carcinoma. Cancer Epidemiol. 36(4), 391-394 (2012).
23 Wang G, Kwan BC, Lai FM, Chow KM, Li PK, Szeto CC. Elevated levels of miR-146a and miR-155 in kidney biopsy and urine from patients with IgA nephropathy. Dis. Markers. 30(4), 171-179 (2011).

24 van Balkom BW, Pisitkun T, Verhaar MC, Knepper MA. Exosomes and the kidney: prospects for diagnosis and therapy of renal diseases. Kidney Int. 80 (11), 1138-1145 (2011).

25 Gildea JJ, Seaton JE, Victor KG et al. Exosomal transfer from human renal proximal tubule cells to distal tubule and collecting duct cells. Clin. Biochem. 47(15), 89-94 (2014).

26 Miranda KC, Bond DT, McKee M et al. Nucleic acids within urinary exosomes/microvesicles are potential biomarkers for renal disease. Kidney Int. 78(2), 191-199 (2010).

27 Sole C, Cortes-Hernandez J, Felip ML, Vidal M, Ordi-Ros J. miR-29c in urinary exosomes as predictor of early renal fibrosis in lupus nephritis. Nephrol. Dial. Transplant. 30(9), 1488-1496 (2015).

28 Etheridge A, Lee I, Hood L, Galas D, Wang K. Extracellular microRNA: a new source of biomarkers. Mutat. Res. 717(1-2), 85-90 (2011).

29 Becker N, Lockwood CM. Pre-analytical variables in miRNA analysis. Clin. Biochem. 46(10-11), 861-868 (2013). 